\title{
A Decomposition Strategy for Optimal Design of a Soda Company Distribution System
}

\author{
J. A. Marmolejo, ${ }^{1}$ I. Soria, ${ }^{2}$ and H. A. Perez ${ }^{2}$ \\ ${ }^{1}$ Faculty of Engineering, Anahuac University, 52786 Mexico City, MEX, Mexico \\ ${ }^{2}$ CADIT, Anahuac University, 52786 Mexico City, MEX, Mexico \\ Correspondence should be addressed to J. A. Marmolejo; jose.marmolejo@anahuac.mx
}

Received 2 May 2015; Revised 9 July 2015; Accepted 28 July 2015

Academic Editor: Pan Liu

Copyright (c) 2015 J. A. Marmolejo et al. This is an open access article distributed under the Creative Commons Attribution License, which permits unrestricted use, distribution, and reproduction in any medium, provided the original work is properly cited.

\begin{abstract}
This work presents a distribution problem of products of a soda bottling company. Commodities are produced at several plants with limited capacity and the demand of distribution centers is satisfied by shipping via cross-docking warehouses. The decomposition strategy is proposed to determine which warehouse needs to be opened to consolidate the demand and by which warehouse each distribution center is served exclusively. The objective is minimizing fixed costs and total transportation costs. The model presented is a mixed-integer programming model with binary variables for which we propose a decomposition strategy based on Benders algorithm. Numerical results show that the proposed strategy can provide the optimal solution of several instances. A large-scale case study based on a realistic company situation is analyzed. Solutions obtained by the proposed method are compared with the solution of full scale problem in order to determine the quality bound and computational time.
\end{abstract}

\section{Introduction}

According to international organisms, Mexico is the second biggest consumer of bottled soda with an average consumption of 160 liters per person a year after the United States, where $94 \%$ of the population consumes these types of drinks. The main point of sale of soda in Mexico is in small stores where $75 \%$ of the sales are carried out, $24 \%$ is in restaurants, and the remaining are in self-service stores [1]. For the company, it is very important to have an effective distribution network that provides the possibility to keep a high level of service to the client at the smallest possible cost; that is, the clients must have products in the opportune moment at minimum cost.

In 2012, the bottling company offered a level of service above $99.6 \%$ (delivery requested/customer request), but in 2013 the first supply problems were presented due to the productive capacity of the plants; this situation originated the specialization of the production lines of the company plants, holding the product readiness in the market at minimum cost; see Figure 1.

For this reason, we proposed a strategy for optimal design of a soda bottling company distribution system based on [1]
The proposed distribution network is constituted by plants, cross-dock warehouses, and distribution centers. Commodities are produced at several plants with limited capacity and the demand of distribution centers is satisfied by shipping via cross-docking warehouses. The problem is to determine which warehouse needs to be opened to consolidate the demand and by which warehouse each distribution center is served exclusively. The objective is minimizing fixed costs and total transportation costs; see Figure 2.

The idea is to establish a network of arcs which enables the flow of products in order to satisfy demand of distribution centers. A proper design can yield better operation levels and cost reductions. In this problem, these reductions can be significant. To the best of our knowledge, the specific model we pose in this study is not addressed in the literature; however, the area of fixed charge network design is closely related.

This paper is organized as follows. In Section 2, we present a brief review of the network design problem. Section 3 contains the specific problem formulation and then Section 4 describes the solution methodology. Section 5 presents computational results of the application of Benders 


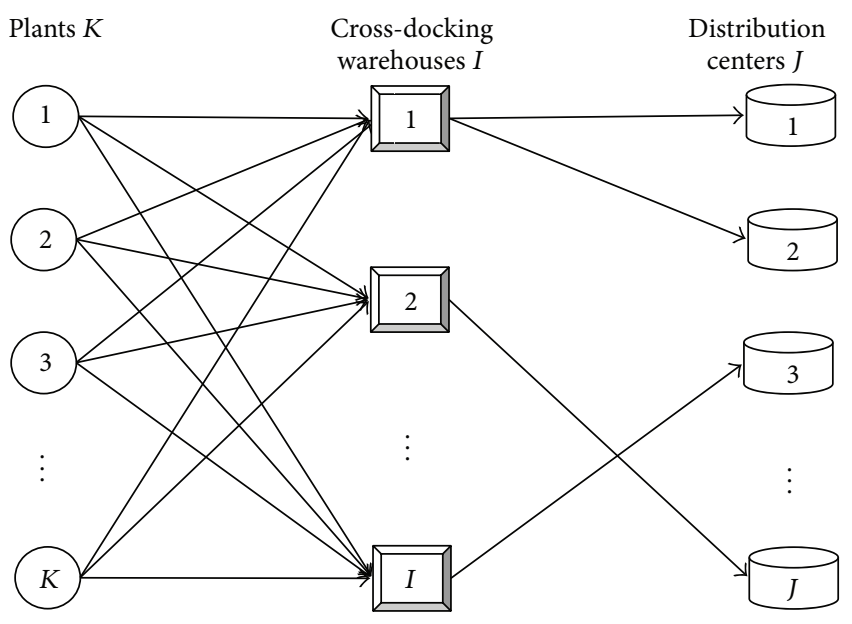

Figure 1: Proposed distribution system.

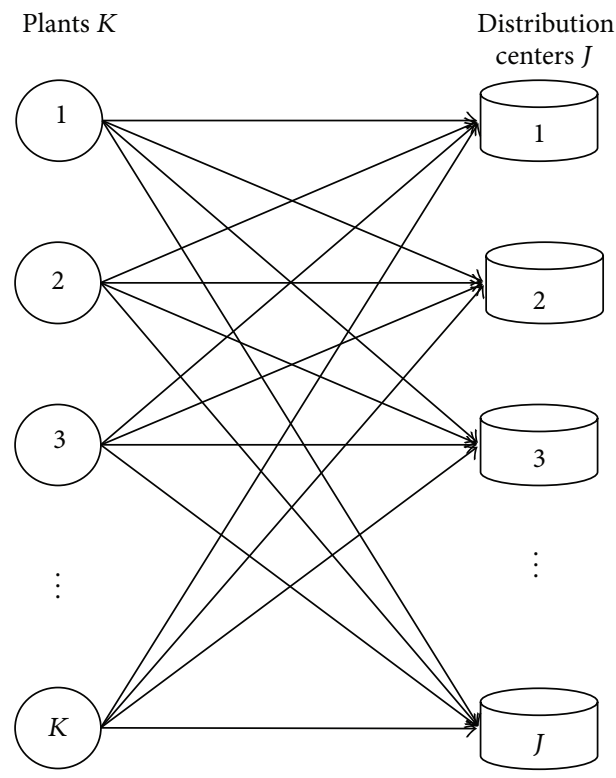

Figure 2: Current distribution system.

Decomposition in several instances. Finally, conclusions are in Section 6.

\section{Literature Review}

Nowadays, companies still pay attention to efficiency because of tactical and operational impact at the executive level; these aspects remain relevant when a new framework is proposed, because all decisions will depend on it to continue the pursuit of all the company objectives. This issue is referred to as supply chain network design problems by most researchers and frequently begins from real-life applications. In this sense, a traditional paper is [2]. They present a new method for the solution of the problem addressing the optimal location of distribution centers between plants and customers. A particular interest involves a study in facility location that is reviewed in [3], where the authors explain some important characteristics, methods, and application in the context of supply chain management. Furthermore, they mentioned that the discrete facility location problems could be categorized as supply chain network design problems. Despite depending on situations, many techniques used in the design of a distribution network are from the research operations area, especially those presented in [4]; some contributions are made to the state of the art that follows this subject for facility locations models. However, several industries are still facing problems related to design of the distribution network because it is a decision linked to improving the level of service, and many other important metrics. Along the same line, in [5], they use a case study related with a model for the distribution network of a region of consumer goods company taking into account several tactical decisions like lead time, credit performance, power, and distribution's reputation. Among various types of problems discussed in supply chain management, facility location has been studied for a long time by some researchers [6]. In [7], the authors follow an objective to propose a novel scheme of distribution. In these investigations, they include a classical facility location problem, and they presented cases of studies solved by appropriate techniques.

Typically, these problems are presented as a mixedinteger programming (MIP) formulation [8, 9]. In [2], the authors develop an algorithm based on Benders Decomposition for solving multicommodity distribution network design problem. Another classical model is presented in [10]. They consider a model to solve a minimization function which includes fixed cost in warehouses and distribution centers and transportation cost for multicommodities from plants to warehouses and finally to customers. Moreover, they consider a tri-echelon, multicommodity system concerning production, distribution, and transportation planning. The authors use a Lagrangian relaxation-based heuristic to provide an effective feasible solution for the problem. Also, they reconsider other different characteristics for solving the main problem of integrated logistics model $[8,11,12]$. In [13], they consider an integrated distribution network design and site selection problem arising in the context of transportation planning faced by the freight-forwarding industry; in this sense, they consider a strategic level multicommodity network design where each commodity is defined by a unique pair of origin and destination points and known required flow amount and other considerations proper to the real problem but using Benders Decomposition for its solution. Similarly, they illustrate the efficiency and the effectiveness of this approach. As in $[14,15]$ also a Benders Decomposition approach is used, first in combination with an intelligent algorithm to improve the time solution for the master problem and then in modified version to exploit the mathematical formulation of the problem in deterministic, multicommodity, single-period contexts, respectively. In [16], the authors exposed that while these production-distribution problems focused to pursue exact solutions efficiently by using optimization software (only for small instances and small dimensions), the main reason to avoid it is because they contain a large number of constraints and variables. Besides, they 
propose heuristics for this bilevel mathematical problem using Stackelberg's equilibrium.

\section{Mathematical Formulation}

Let $K$ be the set of manufacturing plants. An element $k \in K$ identifies a specific plant of the company. Let $I$ be the set of the potential cross-dock warehouses. An element $i \in I$ is a specific cross-dock warehouse. Finally, let $J$ be the set of distribution centers; a specific distribution center is any $j \in J$. Let $\mathbb{Z}$ denote the set of integers $\{0,1\}$.

Parameters are as follows:

$Q_{k}$ : capacity of plant $k$.

$K_{i}$ : capacity of cross-dock warehouse $i$.
$F_{i}$ : fixed costs of opening cross-dock warehouse in location $i$.

$G_{k i}:$ transportation cost per unit of the product from the factories $k$ to cross-dock warehouse $i$.

$C_{i j}$ : cost of shipping the product from the cross-dock $i$ to distribution center $j$.

$d_{j}$ : demand of the distribution center $j$.

Decision Variables. We have the following sets of binary variables to make the decisions about the opening of the cross-dock warehouse, and the distribution for the crossdock warehouse to the distribution center:

$$
\begin{aligned}
& Y_{i}= \begin{cases}1 & \text { if location } i \text { is used as a cross-dock warehouse, } \\
0 & \text { otherwise, }\end{cases} \\
& X_{i j}= \begin{cases}1 & \text { if cross-dock } i \text { supplies the demand of distribution center } j, \\
0 & \text { otherwise. }\end{cases}
\end{aligned}
$$

$W_{k i}$ is the amount of product sent from factory $k$ to crossdock $i$ and is represented by continuous variables.

We can now state the mathematical model as a (P) problem

$$
\min _{W_{k i}, Y_{i}, X_{i j}} Z=\sum_{k \in K} \sum_{i \in I} G_{k i} W_{k i}+\sum_{i \in I} F_{i} Y_{i}+\sum_{i \in I} \sum_{j \in J} C_{i j} d_{j} X_{i j}
$$

subject to the following constraints:

Capacity of the plant:

$$
\sum_{i \in I} W_{k i} \leq Q_{k}, \quad \forall k \in K .
$$

Balance of product:

$$
\sum_{j \in J} d_{j} X_{i j}=\sum_{k \in K} W_{k i}, \quad \forall i \in I .
$$

Single cross-dock warehouse to distribution center:

$$
\sum_{i \in I} X_{i j}=1, \quad \forall j \in J \text {. }
$$

Cross-dock warehouse capacity:

$$
\sum_{j \in J} d_{j} X_{i j} \leq K_{i} Y_{i}, \quad \forall i \in I
$$

Demand of items:

$$
\begin{aligned}
p Y_{i} & \leq W_{k i}, \quad \forall i \in I, \quad \forall k \in K, \\
p & =\min \left\{d_{j}\right\}, \\
W_{k i} & \geq 0, \quad \forall i \in I, \quad \forall k \in K, \\
Y_{i} & \in \mathbb{Z}, \quad \forall i \in I, \\
X_{i j} & \in \mathbb{Z}, \quad \forall i \in I, \forall j \in J .
\end{aligned}
$$

Objective function (2) considers in the first term the cost of shipping the product from the plants $k$ to cross-dock warehouse $i$. The second term contains the fixed cost to open and operate the cross-dock warehouse $i$. The last term incorporates the cost to supply the demand of the distribution center $j$. Constraints (3) imply that all that is produced in plant $k$ does not violate the capacity of plant $k$. Balance constraints (4) ensure that the amount of products that arrive at a distribution center $j$ is the same as that sent to the plants $k$. The demand of each distribution center $j$ will be satisfied by a single cross-dock warehouse $i$, which is achieved by constraints (5). Constraints (6) bound the amount of products that can be sent to a distribution center $j$ from a crossdock warehouse $i$ that has been open. Finally, constraints (7) guarantee that any opened cross-dock warehouse $i$ receives at least the minimum amount of demand for distribution centers $j$. The demand is satisfied by shipping via crossdock warehouse with each distribution center being assigned exclusively to a single cross-dock warehouse. The possible locations for the cross-dock warehouses are given, but the particular facilities to be used will be selected as a result of the minimum total distribution cost. As a result, efficient load consolidation arises as an important opportunity for profitability in this work. To the best of our knowledge, the specific model we pose in this study is not addressed in the literature; however, the area of fixed charge network design is closely related. Our formulation facilitates the use of a Benders Decomposition framework for its solution.

\section{Solution Methodology}

Because of the economic importance of the network design problems and their combinatorial nature, several solution 


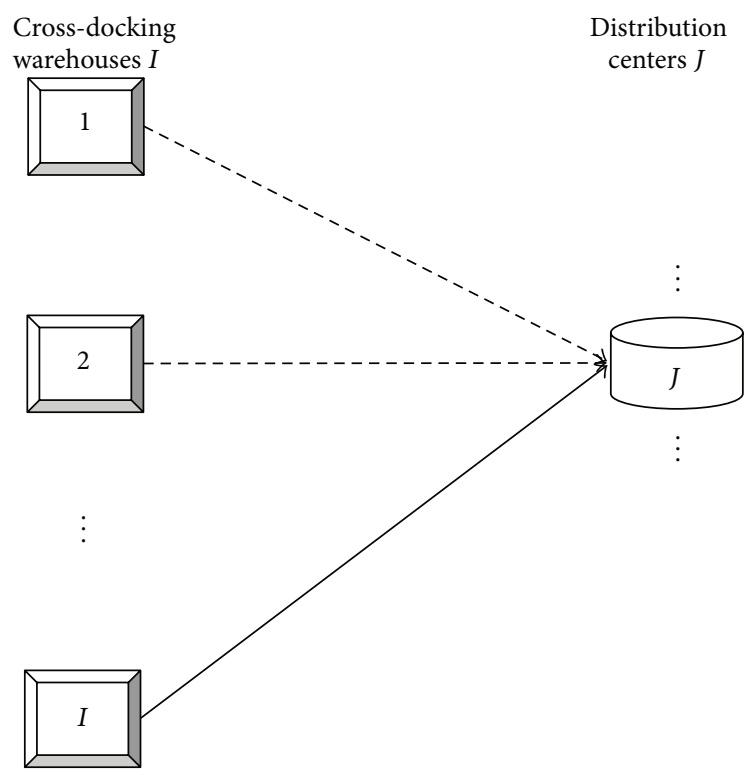

FIGURE 3: One cross-dock warehouse to one distribution center.

methodologies have been developed. In this paper, we describe a decomposition approach for solving the soda bottling company problem. The approach is based on Benders Decomposition, which is one of the most successful solution approaches. Model (2)-(11) presents a structure well-suited for a primal decomposition approach (Benders Decomposition).

This solution method is based on the situation that we can decompose the original problem obtaining several smaller and thus easier to solve subproblems. In this case, Benders Decomposition is used to meet the optimal location of intermediate warehouses between plants and distribution centers. As in [2], this model proposes that no customer zone is allowed to deal with more than one cross-dock warehouse, since $X_{i j}$ must be 0 or 1 and not fractional. Additionally, we can mention that the real case presented has a large number of binary variables compared to the number of constraints. Thus, the Benders Decomposition is the better way to solve the optimal design of the distribution system, because it exploits the special structure of the original problem. In Figure 1 a graphical representation of the proposed distribution system is shown. This graphical illustration is different from the one presented in Figure 2. The difference between the current system and the proposed is because the demand is satisfied by shipping via cross-dock warehouse with each distribution center, which is assigned exclusively to a single cross-dock warehouse; see Figure 3.

4.1. Benders Decomposition. The optimal design of a soda bottling company distribution system is a problem that has a large number of complicating binary variables and thus involves a large CPU time to find an optimal integer solution; for this reason, we applied Benders Decomposition framework [17]. This method projects problem (2)-(11) onto the space defined by the binary variables $X_{i j}$ and $Y_{i}$. The original problem $\mathrm{P}$ is decomposed into two different problems: a restricted master problem and Benders subproblem.
The subproblem (SP) is a dual lineal problem (LP) of $\mathrm{P}$ and is obtained by fixing the variables to either 0 or 1 . Benders subproblem provides an upper bound of the original problem. Let (SP) be the dual problem of $\mathrm{P}$ and the dual variables associated with constraints (3), (4), and (7), respectively. We have the following:

Subproblem (SP):

$$
\begin{gathered}
\max _{\omega_{k}, \alpha_{i} \beta_{i k}} \Phi=\sum_{k \in K} Q_{k} \omega_{k}+\sum_{i \in I} \sum_{j \in J}\left(d_{j} \bar{X}_{i j}\right) \alpha_{i}+\sum_{k \in K} \sum_{i \in I} \bar{Y}_{i} \beta_{i k}, \\
\omega_{k}+\alpha_{i}+\beta_{i k} \leq G_{k i}, \quad \forall i \in I, \quad \forall k \in K, \\
\omega_{k} \leq 0,
\end{gathered}
$$

$\alpha_{i}$ unrestricted,

$$
\beta_{i k} \geq 0 .
$$

Relaxed Master Problem (RMP):

$$
\begin{aligned}
& \min _{Y_{i}, X_{i j}} \Omega \text {, } \\
& \Omega \geq \sum_{i \in I} F_{i} Y_{i}+\sum_{i \in I} \sum_{j \in J}\left(C_{i j} d_{j} X_{i j}\right) \bar{\omega}_{k}+\sum_{i \in I} \sum_{k \in K} Y_{i} \bar{\beta}_{i k} \\
& +\sum_{i \in I} \sum_{j \in J}\left(d_{j} X_{i j}\right) \bar{\alpha}_{i}, \\
& \sum_{i \in I} F_{i} Y_{i}+\sum_{i \in I} \sum_{j \in J}\left(C_{i j} d_{j} X_{i j}\right) \bar{\omega}_{k}+\sum_{i \in I} \sum_{k \in K} Y_{i} \bar{\beta}_{i k} \\
& +\sum_{i \in I} \sum_{j \in J}\left(d_{j} X_{i j}\right) \bar{\alpha}_{i} \leq 0, \\
& \sum_{i \in I} X_{i j}=1, \quad \forall j \in J, \\
& \sum_{i \in I} X_{i j} \leq 0, \quad \forall j \in J, \\
& \sum_{j \in J} d_{j} X_{i j} \leq K_{i} Y_{i}, \quad \forall i \in I, \\
& \sum_{j \in J} d_{j} X_{i j} \leq 0, \quad \forall i \in I, \\
& Y_{i}, X_{i j} \in\{0,1\} .
\end{aligned}
$$

The master problem is an integer problem and its variables are considered as complicating variables. To determine the values of these variables, the Benders master problem must be solved. Since the number of primal cuts is too large, Benders [17] proposed to solve a Relaxed Master Problem (RMP), by taking only a subset or Benders cuts, and to generate these cuts, one by one, in each iteration of the algorithm. Relaxed Master Problem provides feasible solutions through Benders optimality cuts (14) because they are based on optimality conditions of the subproblem and through valid constraints called feasibility cuts in every iteration of the algorithm. Feasibility cuts (15), (16), (17), (18), and (19) enforce necessary conditions for feasibility of the 
TABLE 1: Comparison of the instances.

\begin{tabular}{lcccccc}
\hline Instance & $K$ & $I$ & $J$ & Continuous variables & Binary variables & Constraints \\
\hline INST 0 & 2 & 2 & 2 & 4 & 20 & 90 \\
INST 1 & 4 & 5 & 17 & 17 & 40 & 180 \\
INST 2 & 4 & 10 & 40 & 150 & 1025 & 51 \\
INST 3 & 6 & 25 & 254 & 2464 & 14280 & 121 \\
INST-R CASE & 44 & 56 & & & & 466 \\
\hline
\end{tabular}

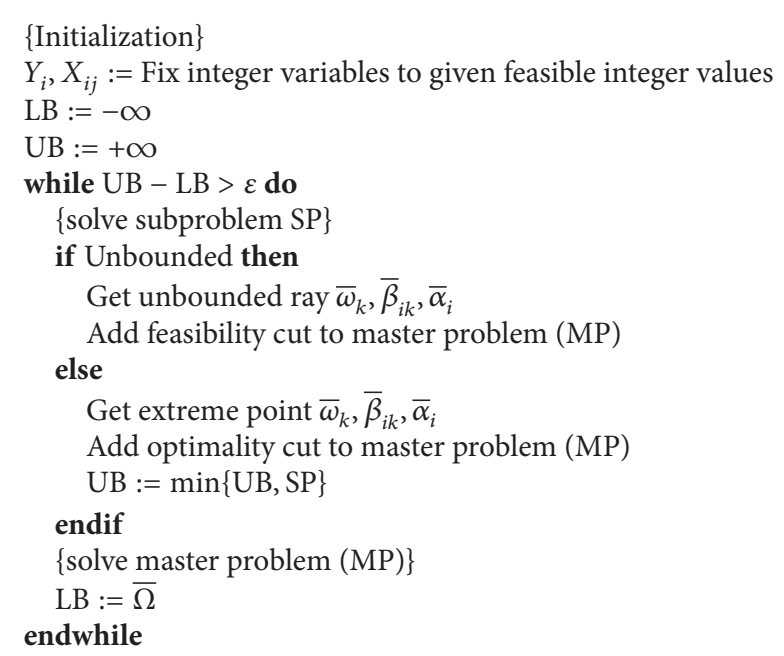

Procedure 1: Benders Decomposition $\left(Y_{i}, X_{i j}, \omega_{k}, \beta_{i k}, \alpha_{i}\right)$.

primal subproblem. Master problem provides a lower bound of the original problem $(\mathrm{P})$.

Benders Algorithm. A brief summary of the algorithm is given in Procedure 1 for completeness.

\section{Computational Experience}

The full scale model and the decomposition strategy proposed were implemented in GAMS [18] using the solver CPLEX [19] for MIP and LP problems (master problem and Benders subproblem). All mathematical models were carried out on an AMD Phenom II N970 Quad-Core with a $2.2 \mathrm{GHz}$ processor and $4 \mathrm{~GB}$ RAM. Because the major difficulty of Benders method is the solution of master problem, and because [2] suggests that the Benders master problem should not be solved to optimality, we set GAMS parameter OPTCR at 0.0015 ; that is, the relative termination tolerance is within $0.15 \%$ of the best possible solution. In the first iteration of algorithm, we fixed all integer variables to 1 . Additionally, the size of all MIP models was reduced through presolver phase of CPLEX. Benders algorithm stops when the values of lower bound and upper bound are equal, except for a small tolerance $\varepsilon=0.15 \%$ :

$$
\varepsilon=\left[\frac{(\mathrm{UB}-\mathrm{LB})}{\mathrm{UB}}\right] \cdot 100 \% .
$$

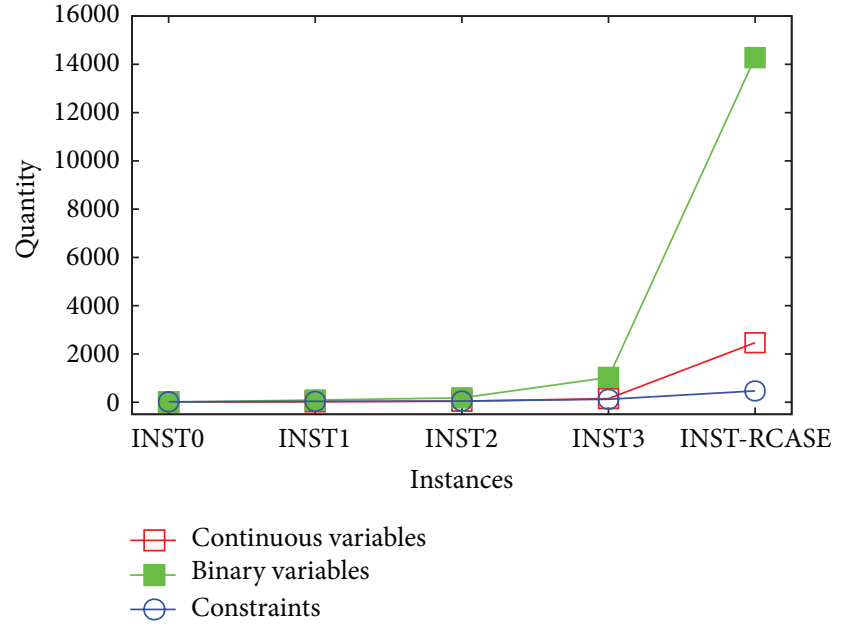

FIGURE 4: Model complexity.

To test the efficiency in terms of CPU time and quality of our solution method, we solved instances with data having demand distribution characteristics that reflect real applications. We generated randomly four instances with different number of plants, cross-dock warehouses, and distribution centers. Additionally, we solved a realistic case of a soda bottling company. Table 1 indicates the specific size of each instance.

The model complexity of each instance can be seen in Figure 4. This figure shows the increase in problem size in terms of the number of constraints, continuous variables, and binary variables.

5.1. Results. Benders Decomposition described in the previous section was used to design the optimal distribution system for the soda bottling company. Because the amount of memory and the computational effort needed to solve the instances grow significantly with the number of variables and constraints, we propose a decomposition strategy based on Benders Decomposition. The comparison results demonstrate that the $\varepsilon$-optimal solution is very close to the optimal solution (GAMS-CPLEX Solution).

Table 2 illustrates that the comparison results demonstrate that the CPU time of proposed decomposition strategy is less than direct solution with GAMS-CPLEX. The GAP is less than $0.2 \%$ :

$$
\begin{aligned}
& \text { GAP } \\
& =\left[\frac{(\text { GAMS Solution }- \text { Benders Decomposition Solution })}{\text { GAMS Solution }}\right] \\
& \quad \cdot 100 \% .
\end{aligned}
$$


TABle 2: Performance of Benders Decomposition and direct solution of full scale problem.

\begin{tabular}{|c|c|c|c|c|c|c|}
\hline Instance & $\begin{array}{c}\text { Opt. value } \\
\text { GAMS-CPLEX }\end{array}$ & $\begin{array}{l}\text { CPU time } \\
\text { (sec.) }\end{array}$ & LB & UB & $\begin{array}{l}\text { GAP } \\
(\%) \\
\end{array}$ & $\begin{array}{c}\text { CPU time } \\
\text { (sec.) }\end{array}$ \\
\hline INST 0 & 1270 & 1.5 & 1270 & 1270 & $<0.2$ & 0.5 \\
\hline INST 1 & 179170 & 2.5 & 179168 & 179168 & $<0.2$ & 1.5 \\
\hline INST 2 & 485140 & 3.5 & 485142 & 485142 & $<0.2$ & 2.5 \\
\hline INST 3 & $1.00 E+07$ & 930 & $1.00 E+07$ & $1.00 E+07$ & $<0.2$ & 630 \\
\hline INST-R CASE & $8.45 E+10$ & 4160 & $8.43 E+10$ & $8.43 E+10$ & 0.2 & 2160 \\
\hline
\end{tabular}
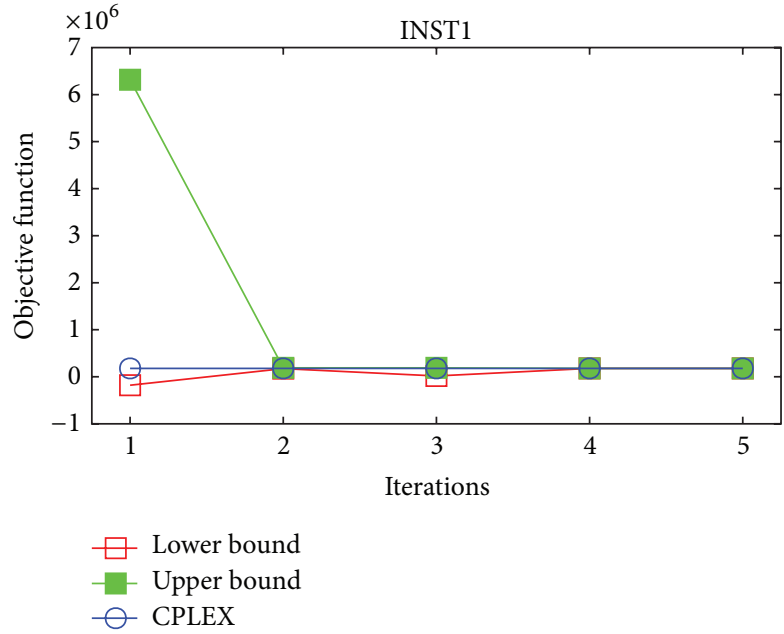

FIGURE 5: Benders versus full scale solution.

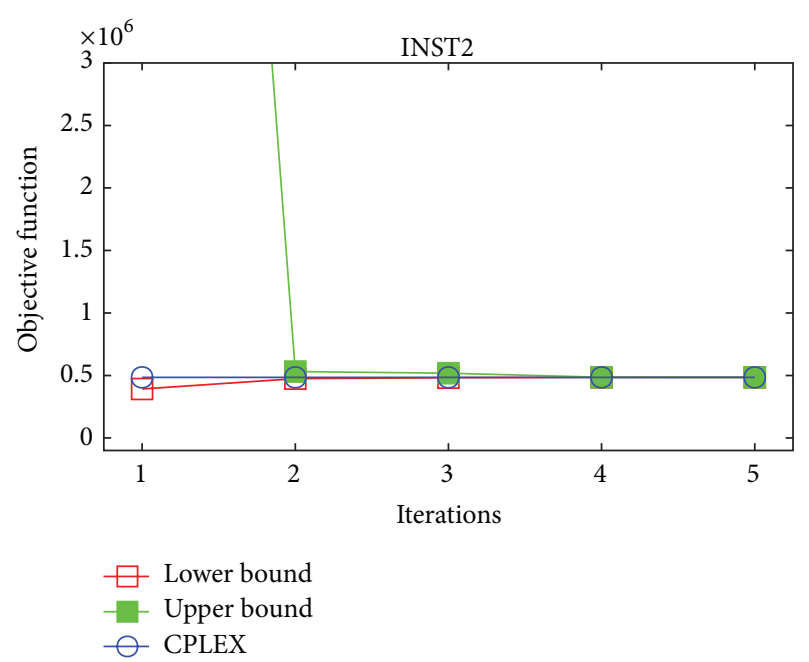

FIGURE 6: Benders versus full scale solution.

The progression of upper bound and lower bound values over the iterations around the optimum in all instances is plotted in Figures 5, 6, 7, and 8. Clearly, the use of feasibility cuts is very effective in speeding up convergence and they affect the quality of both the upper and the lower bounds. In our numerical studies, we observe similar high performance in convergence with feasibility cuts in other instances. For all the instances solved, tight lower and upper bounds were

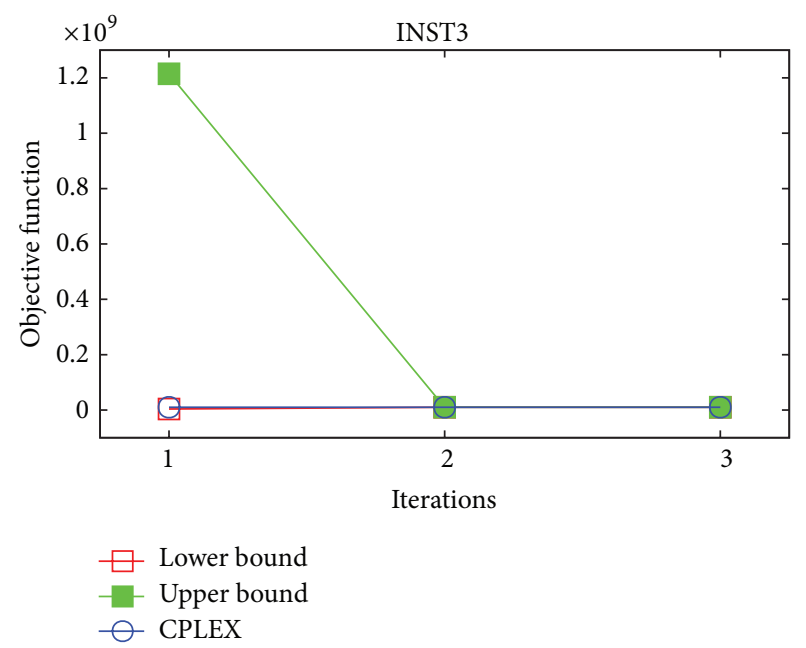

Figure 7: Benders versus full scale solution.

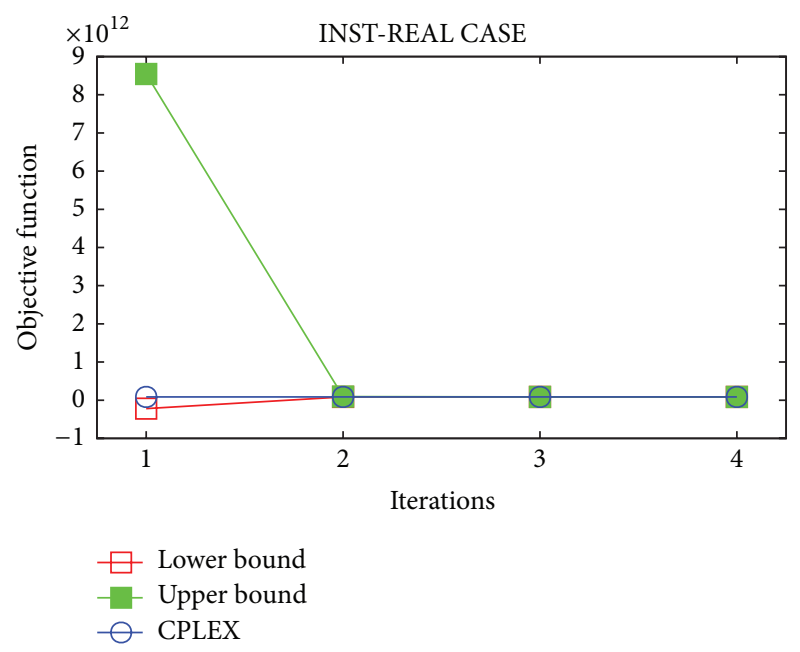

FIGURE 8: Benders versus full scale solution.

obtained with a small GAP in just a few iterations of the proposed algorithm.

These measures reported show that the performance of the Benders Decomposition seems to be indifferent to its size when feasibility cuts are implemented. The results of the experiments reported in Table 2 show that the proposed Benders procedure produces very good feasible solutions compared to the optimal/best available ones generated by CPLEX in significantly less CPU time. 


\section{Conclusions}

In this paper, we present an optimal design of a soda bottling company distribution system problem. We proposed a decomposition strategy that shows acceptable convergence properties. In all instances, the number of iterations required to get convergence is less than five. The feasibility cuts allow reaching an $\varepsilon$-optimal solution. The total CPU time required to solve the large-scale case study based on the realistic company situation was less than 2200 seconds. This instance has 14280 binary variables, 2464 continuous variables, and 466 constraints. The proposed decomposition strategy was shown to be very efficient for the case study. For this class of problems, global optimality is not guaranteed in a reasonable time, but the solution through Benders Decomposition provides good feasible solutions and good bounds to the optimum.

Moreover, it is important to mention that the main objective was to compare the CPU effort and the quality bounds of the full scale solution through a commercial solver and the proposed decomposition strategy. We can finally conclude that we could propose a strategy for designing the distribution system of a soda bottling company in competitive CPU times. The solutions obtained are close to the optimal values reported by commercial optimizers. This ensures having a flexible and scalable solution tool when the company decides to increase the number of its facilities.

\section{Conflict of Interests}

The authors declare that there is no conflict of interests regarding the publication of this paper.

\section{References}

[1] I. Soria, Rediseño de la cadena de abastecimiento de un grupo embotellador de bebidas [M.S. thesis], ITESM Campus Toluca, Toluca, Mexico, 2008.

[2] A. M. Geoffrion and G. W. Graves, "Multicommodity distribution system design by benders decomposition," Management Science, vol. 20, no. 5, pp. 822-844, 1974.

[3] M. T. Melo, S. Nickel, and F. S. da-Gama, "Facility location and supply chain management-a review," European Journal of Operational Research, vol. 196, no. 2, pp. 401-412, 2009.

[4] A. Klose and A. Drexl, "Facility location models for distribution system design," European Journal of Operational Research, vol. 162, no. 1, pp. 4-29, 2005.

[5] A. Cintron, A. R. Ravindran, and J. A. Ventura, "Multi-criteria mathematical model for designing the distribution network of a consumer goods company," Computers \& Industrial Engineering, vol. 58, no. 4, pp. 584-593, 2010.

[6] M. S. Jabalameli, B. Bankian Tabrizi, and M. Javadi, "Capacitated facility location problem with variable coverage radius in distribution system," International Journal of Industiral Engineering \& Production Research, vol. 21, no. 4, pp. 231-237, 2010.

[7] P. N. Thanh, O. Péton, and N. Bostel, "A linear relaxation-based heuristic approach for logistics network design," Computers \& Industrial Engineering, vol. 59, no. 4, pp. 964-975, 2010.

[8] V. Jayaraman and H. Pirkul, "Planning and coordination of production and distribution facilities for multiple commodities,"
European Journal of Operational Research, vol. 133, no. 2, pp. 394-408, 2001.

[9] H. Sadjady and H. Davoudpour, "Two-echelon, multi-commodity supply chain network design with mode selection, leadtimes and inventory costs," Computers and Operations Research, vol. 39, no. 7, pp. 1345-1354, 2012.

[10] H. Pirkul and V. Jayaraman, "Production, transportation, and distribution planning in a multi-commodity tri-echelon system," Transportation Science, vol. 30, no. 4, pp. 291-302, 1996.

[11] V. Jayaraman, "An efficient heuristic procedure for practicalsized capacitated warehouse design and management," Decision Sciences, vol. 29, no. 3, pp. 729-745, 1998.

[12] H. Pirkul and V. Jayaraman, "A multi-commodity, multi-plant, capacitated facility location problem: formulation and efficient heuristic solution," Computers \& Operations Research, vol. 25, no. 10, pp. 869-878, 1998.

[13] H. Üster and H. Agrahari, "A Benders decomposition approach for a distribution network design problem with consolidation and capacity considerations," Operations Research Letters, vol. 39, no. 2, pp. 138-143, 2011.

[14] W. Jiang, L. Tang, and S. Xue, "A hybrid algorithm of tabu search and benders decomposition for multi-product production distribution network design," in Proceedings of the IEEE International Conference on Automation and Logistics (ICAL '09), pp. 79-84, August 2009.

[15] H. M. Bidhandi, R. M. Yusuff, M. M. H. M. Ahmad, and M. R. Abu Bakar, "Development of a new approach for deterministic supply chain network design," European Journal of Operational Research, vol. 198, no. 1, pp. 121-128, 2009.

[16] J.-F. Camacho-Vallejo, R. Muñoz-Sánchez, and J. L. GonzálezVelarde, "A heuristic algorithm for a supply chain's productiondistribution planning," Computers \& Operations Research, vol. 61, pp. 110-121, 2015.

[17] J. F. Benders, "Partitioning procedures for solving mixedvariables programming problems," Computational Management Science, vol. 2, no. 1, pp. 3-19, 2005.

[18] A. Brooke, D. Kendrick, and A. Meeraus, GAMS: A User's Guide, Boyd \& Fraser Publishing Company, 1998.

[19] GAMS Development Corporation, GAMS: The Solver Manuals; CONOPT; CPLEX; DICOPT; LAMPS; MILES; MINOS; OSL; PATH; XA; ZOOM, GAMS Development Corporation, 1994. 


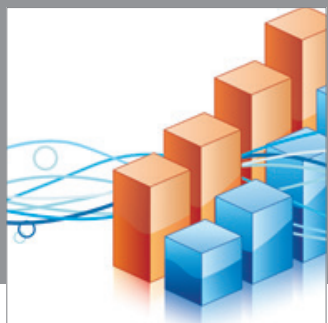

Advances in

Operations Research

mansans

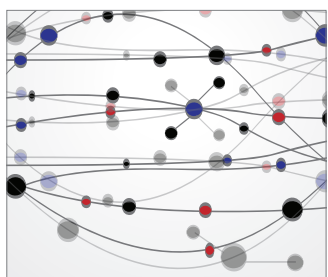

The Scientific World Journal
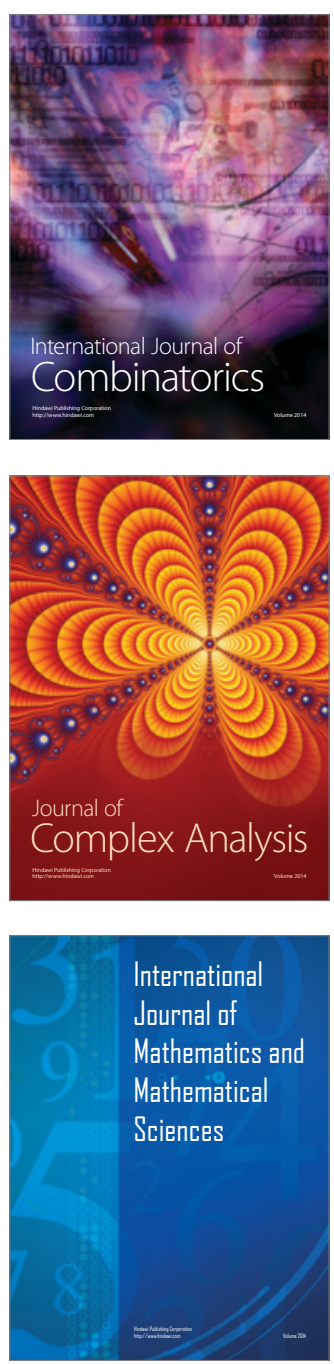
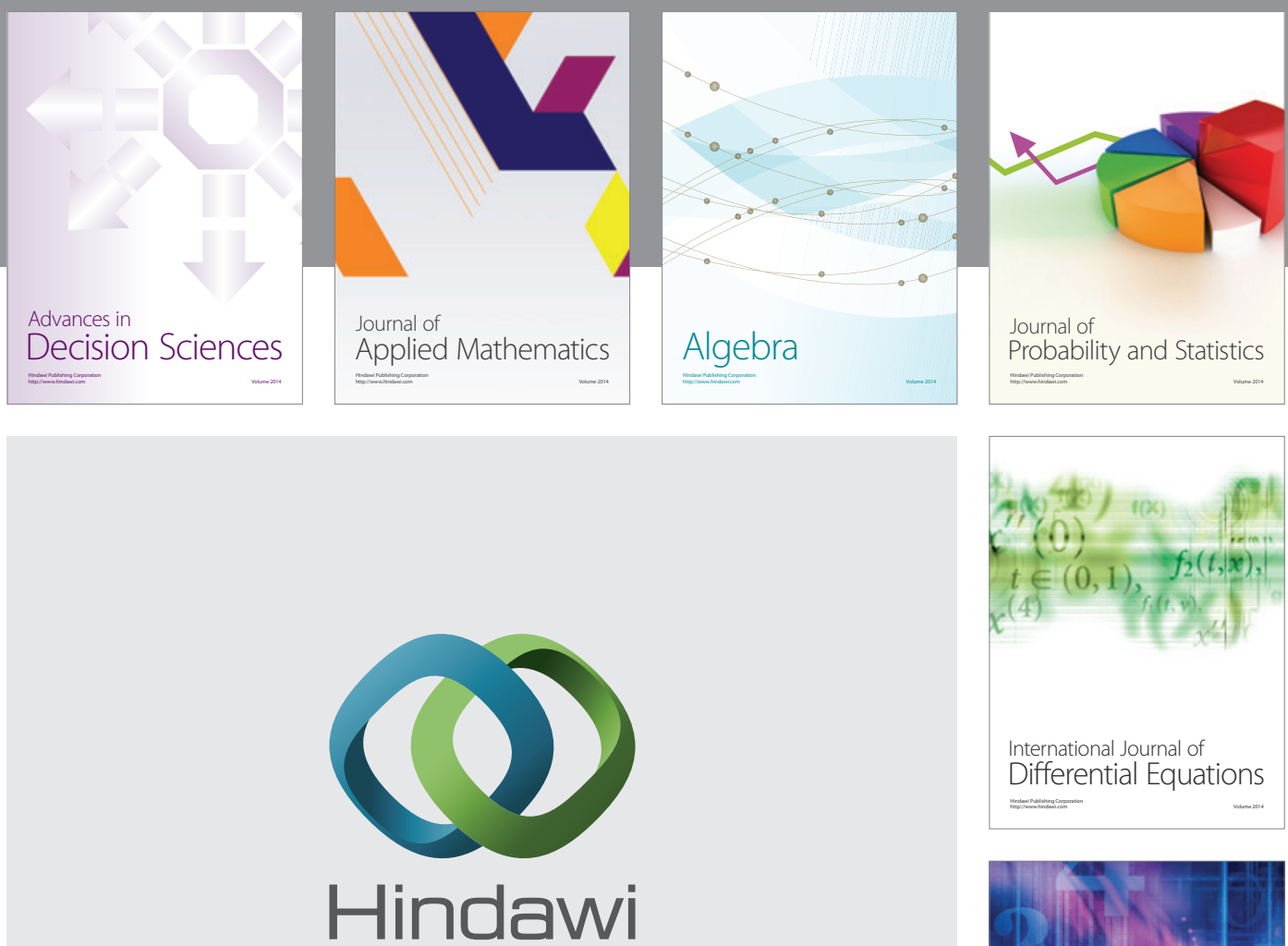

Submit your manuscripts at http://www.hindawi.com
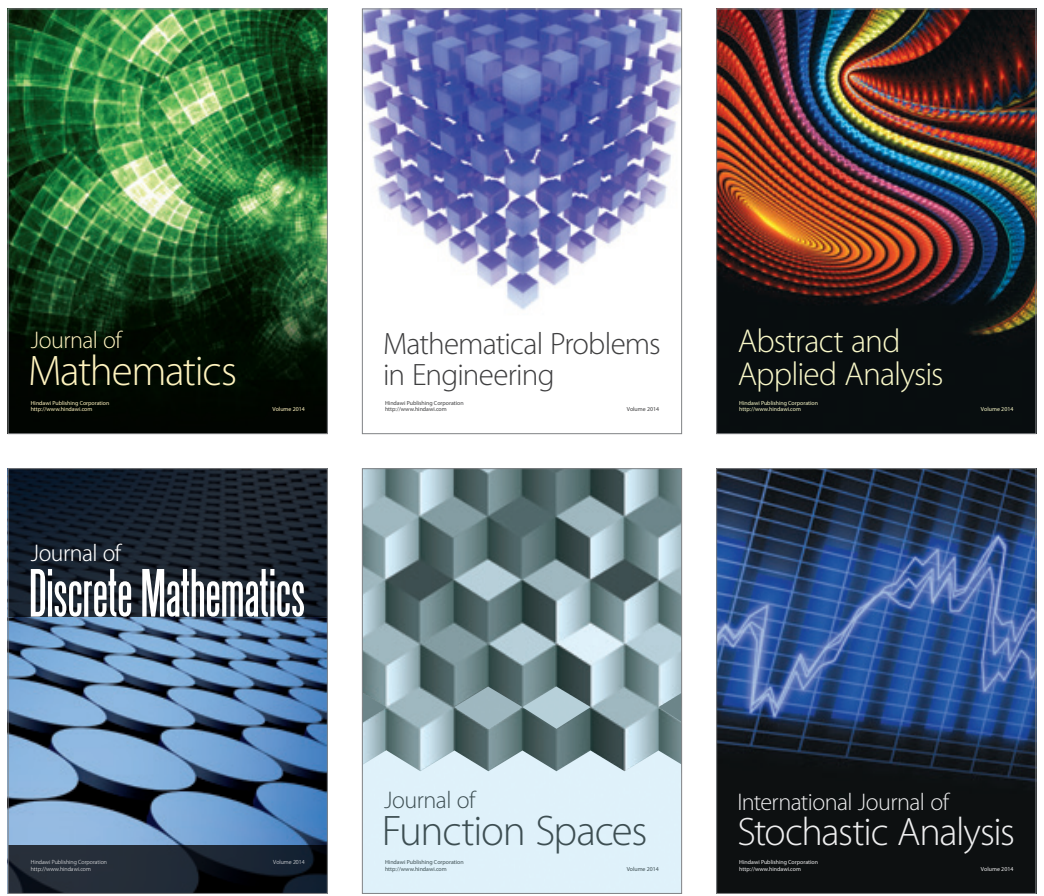

Journal of

Function Spaces

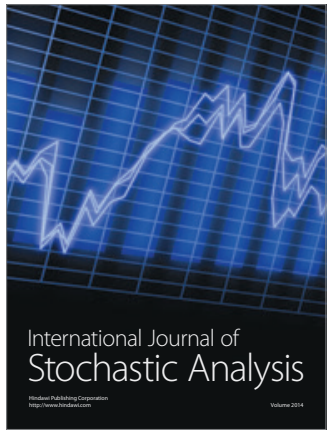

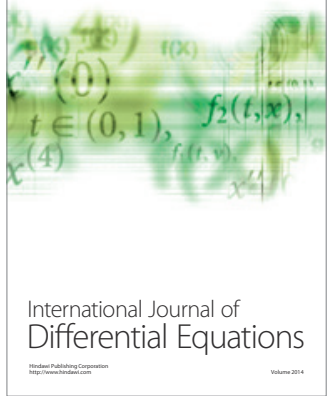
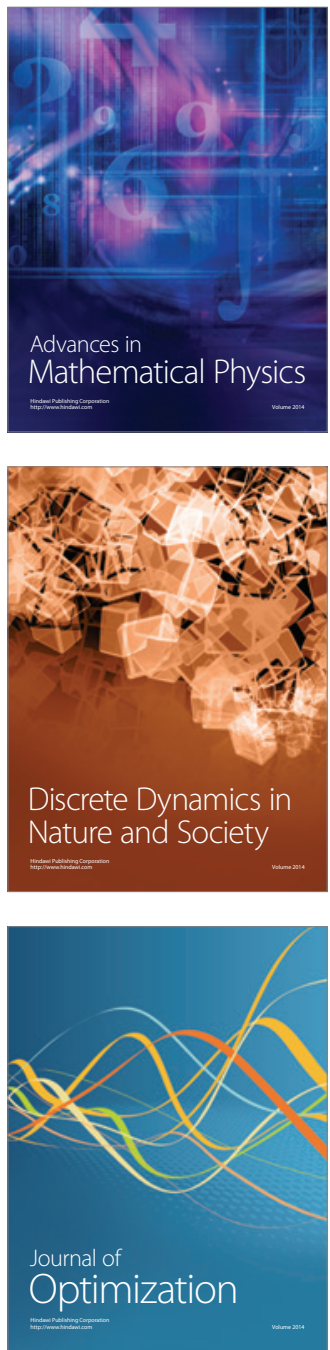